\title{
A Weighted Least Squares Approach to the Design of FIR Filters Synthesized Using the Modified Frequency Response Masking Structure
}

\author{
W. R. Lee, L. Caccetta, K. L. Teo, Senior Member, IEEE, and V. Rehbock
}

\begin{abstract}
This paper presents an application of the weighted least squares (WLS) method to the design of sharp linear phase finite-impulse response (FIR) digital filters synthesized using a modified frequency-response masking (FRM) structure. In our approach, the original minimax design problem is converted into a WLS problem. The WLS problem is highly nonlinear with respect to the coefficients of the filter. However, it can be decomposed into four linear least squares (LS) problems, each of which can be solved analytically. The design problem is then solved iteratively by using an alternating variable approach. The effectiveness of the method is demonstrated through solving a low-pass linear phase sharp FIR digital filter example.
\end{abstract}

Index Terms-Frequency-response masking (FRM), minimax error, weighted least squares (WLS), alternating variable approach.

\section{INTRODUCTION}

$\mathbf{T}$ HE frequency-response masking approach is one of the most efficient techniques to synthesize linear-phase finiteimpulse response (FIR) digital filters with sharp transition bands [1]-[4]. This approach produces filters with very sparse coefficients and hence reduces the number of multipliers and adders required when compared with those produced by conventional direct design methods. The basic structure of a filter synthesized using the frequency-response masking (FRM) technique is shown in Fig. 1. If $F(z)$ is the $z$-transform transfer function of the system, then, from Fig. 1

$$
F(z)=F_{a}\left(z^{M}\right) F_{m a}(z)+\left\{z^{\frac{-M(l-1)}{2}}-F_{a}\left(z^{M}\right)\right\} F_{m c}(z) .
$$

Here, $F_{a}\left(z^{M}\right)$ is the interpolated band-edge shaping filter, which can be obtained by replacing each delay element of a prototype filter, $F_{a}(z)$, by $M$ delay elements. $l$ is the filter length of $F_{a}(z) . F_{m a}(z)$ and $F_{m c}(z)$ are two masking filters. Let $w_{p}$ and $w_{s}$ be the band edges of $F(z)$. Then, the transition width of $F_{a}(z)$ is $M\left(w_{s}-w_{p}\right)$, which increases with $M$. Hence, the complexity of $F_{a}(z)$ decreases with increasing

Manuscript received May 11, 2004. This work was supported by the Western Australian Center of Excellence in Industrial Optimization. The work of K. L. Teo was supported by the Center for Multimedia Signal Processing of the Department of Electronics and Information Technology, The Hong Kong Polytechnic University Hung Hom, Kowloon, Hong Kong, and by the Research Grant Council of Hong Kong under Grant Ployu5247/04E. This paper was recommended by Associate Editor Y. Lian.

The authors are with the Western Australian Centre of Excellence in Industrial Optimization, Department of Mathematics \& Statistics, Curtin University of Technology, Perth 6845, Australia.

Digital Object Identifier 10.1109/TCSII.2005.862282

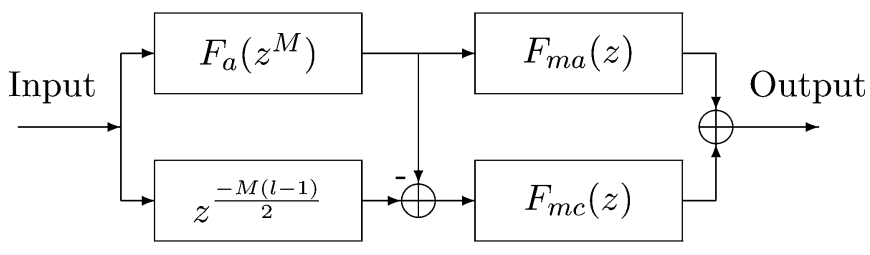

Fig. 1. Basic structure used in the FRM technique.

$M$. However, the sum of the transition widths of $F_{m a}(z)$ and $F_{m c}(z)$ is $(1 / M)$, which is decreasing when $M$ increases. If $M$ is very large, both $F_{m a}(z)$ and $F_{m c}(z)$ become sharp filters. Recently, several modified FRM structures, such as those reported in [5]-[16] have been introduced. In particular, the approach proposed in [12] is to replace the band-edge shaping filter $F_{a}\left(z^{M}\right)$ with an interpolated FIR (IFIR) filter (for a definition, see [17]-[19]) so as to form an IFIR-FRM structure. With this new structure, the requirements on the masking filters are relaxed. However, the drawback of this approach is that the filter length of one of the masking filters becomes excessively long, which is not optimal from a structural point of view. This is because minimum complexity is achieved (see [2], [20]) only when the transition widths (or complexity) of the two masking filters are equal. To address this problem, the IFIR-FRM structure proposed in [12] is further improved in [11]. First, the IFIR-based band-edge shaping filter is generalized to an IFIR alike filter. Secondly, an additional filtering stage is inserted between the band-edge shaping filter and the masking filters so as to decouple the masking filters from the band-edge shaping filter. Hence, the complexity of the overall filter is reduced significantly. However, it is noted that the subfilters are essentially optimized separately in these papers.

This paper introduces a joint optimization method for the design of linear-phase FIR digital filters synthesized using the modified FRM structure introduced in [11]. The method is based on the weighted least squares (WLS) approach. The original least squares (LS) problem is decomposed into four simple linear LS problems. Each of these linear LS problems can be solved analytically. The WLS problem is then solved iteratively by alternately solving each of these four linear LS problems with appropriately updated weighting functions.

\section{PROBLEM}

The modified structure of an FIR filter synthesized using the FRM technique, introduced in [11], is shown in Fig. 2. If $F(z)$ 


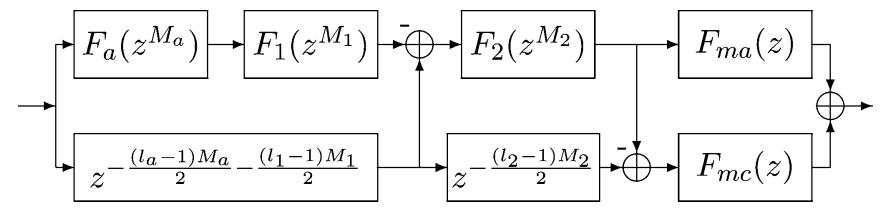

Fig. 2. The Modified FRM structure.
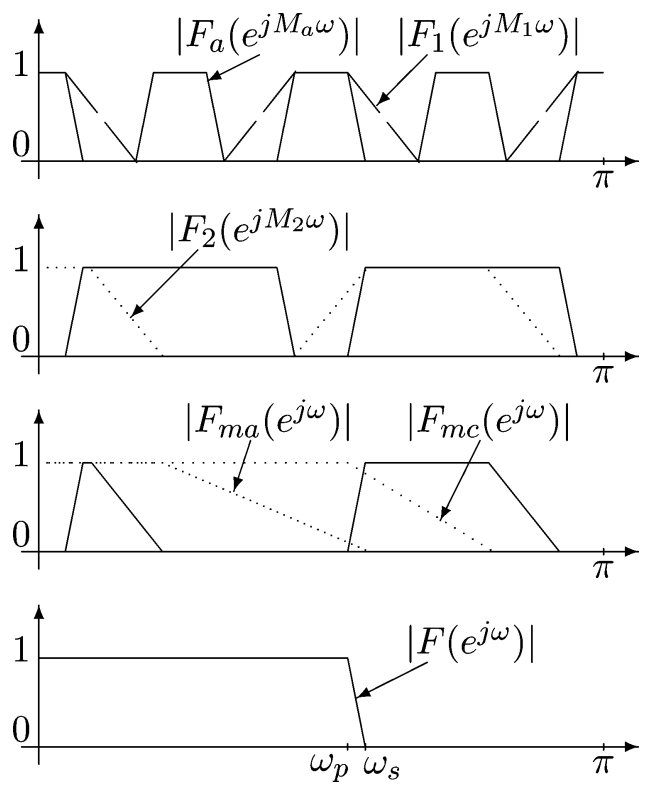

Fig. 3. Frequency responses of (a) $F_{a}\left(z^{M_{a}}\right)$ and $F_{1}\left(z^{M_{1}}\right)$; (b) $F_{2}\left(z^{M_{2}}\right)$; (c) $F_{m a}(z)$ and $F_{m c}(z) ;(d) F(z)$.

is the $z$-transform transfer function of the system, then, from Fig. 2

$$
F(z)=G_{1}(z) F_{m a}(z)+G_{2}(z) F_{m c}(z)
$$

where

$$
\begin{aligned}
G_{1}(z) & =G_{3}(z) F_{2}\left(z^{M_{2}}\right) \\
G_{3}(z) & =z^{-\frac{\left(l_{a}-1\right) M_{a}}{2}-\frac{\left(l_{1}-1\right) M_{1}}{2}}-F_{a}\left(z^{M_{a}}\right) F_{1}\left(z^{M_{1}}\right) \\
G_{2}(z) & =z^{-\frac{\left(l_{a}-1\right) M_{a}}{2}-\frac{\left(l_{1}-1\right) M_{1}}{2}-\frac{\left(l_{2}-1\right) M_{2}}{2}}-G_{1}(z) \\
F_{a}\left(z^{M_{a}}\right) & =\sum_{n=0}^{l_{a}-1} h_{a}(n) z^{-n M_{a}} \\
F_{1}\left(z^{M_{1}}\right) & =\sum_{n=0}^{l_{1}-1} h_{1}(n) z^{-n M_{1}} \\
F_{2}\left(z^{M_{2}}\right) & =\sum_{n=0}^{l_{2}-1} h_{2}(n) z^{-n M_{2}} \\
F_{m a}(z) & =\sum_{n=0}^{l_{m a}-1} h_{m a}(n) z^{-n} \\
F_{m c}(z) & =\sum_{n=0}^{l_{m c}-1} h_{m c}(n) z^{-n} .
\end{aligned}
$$

The frequency response magnitudes $\left|F_{a}\left(e^{j w M_{a}}\right)\right|$, $\left|F_{1}\left(e^{j w M_{1}}\right)\right|,\left|F_{2}\left(e^{j w M_{2}}\right)\right|,\left|F_{m a}\left(e^{j w}\right)\right| \quad$ and $\left|F_{m c}\left(e^{j w}\right)\right|$, of these filters are shown in Fig. 3. If $G_{1}(z) F_{m a}(z)$ and $G_{2}(z) F_{m c}(z)$ have the same phase delay, the resulting frequency response magnitude, $\left|F\left(e^{j w}\right)\right|$, of $F(z)$ is shown in Fig. 3(d). The transition width of $F(z)$ is narrower than that of $F_{a}(z)$ by a factor of $M_{a}$. With this new structure, the restriction on $M_{a}$ to be a multiple of $M_{1}$, which is required for an IFIR-FRM approach [12], is relaxed. Furthermore, an additional decoupling filter $F_{2}\left(z^{M_{2}}\right)$ is inserted to balance the complexities of the two masking filters. The requirement on the masking filters $F_{m a}(z)$ and $F_{m c}(z)$ is, therefore, further relaxed (For details, see [11]).

Given the passband edge $w_{p}$, the stopband edge $w_{s}$, the permitted maximum passband ripple $\delta_{p}$, and the stopband ripple $\delta_{s}$ of a desired filter, the interpolation ratios $M_{a}, M_{1}$, and $M_{2}$ for the subfilters $F_{a}, F_{1}$, and $F_{2}$, respectively, can be optimized (for details, see [11]). The band edges for the subfilters $F_{a}, F_{1}, F_{2}, F_{m a}$ and $F_{m c}$ can be derived using the equations in [1], [11], [20]. Therefore, the general problem of designing an FIR filter synthesized using the modified FRM structure introduced in [11] may be formulated as: Given the band edges $w_{p}$ and $w_{s}$, the permitted maximum band ripples $\delta_{p}$ and $\delta_{s}$, and the interpolation ratios $M_{a}, M_{1}$ and $M_{2}$, find, respectively, the filter lengths $l_{a}, l_{1}, l_{2}, l_{m a}$ and $l_{m c}$ of the subfilters of the system, and coefficients $\boldsymbol{h}_{a}, \boldsymbol{h}_{1}, \boldsymbol{h}_{2}, \boldsymbol{h}_{m a}$ and $\boldsymbol{h}_{m c}$ of the subfilters $F_{a}, F_{1}, F_{2}, F_{m a}$ and $F_{m c}$, such that the maximum error, $E(w)$, is minimized over the whole region, where $E(w)$ is defined by

$$
E(w)=W(w)|D(w)-F(w)| .
$$

Here, $W(w)$ and $D(w)$ are the frequency weighting function and the desired response of the system, respectively. In the passband, $W(w)$ and $D(w)$ are equal to one. In the stopband, $W(w)$ is equal to $\delta_{p} / \delta_{s}$ and $D(w)$ is equal to zero.

This minimax problem is to be solved as a WLS problem. More specifically, we minimize the objective function

$$
\epsilon=\sum_{w \in \Xi} B^{2}(w)|E(w)|^{2}
$$

where $\Xi$ is a dense set of frequencies uniformly distributed in $\left[0, w_{p}\right] \cup\left[w_{s}, 2 \pi\right]$, and $B(w)$ is a weighting function. Let this WLS problem be referred to as Problem $(P)$.

\section{SOLUTION METHOD}

Suppose that the filter lengths $l_{a}, l_{1}, l_{2}, l_{m a}, l_{m c}$, of the subfilters are determined. Then, Problem $(P)$ reduces to finding the coefficients $\boldsymbol{h}_{a}, \boldsymbol{h}_{1}, \boldsymbol{h}_{2}, \boldsymbol{h}_{m a}$ and $\boldsymbol{h}_{m c}$ of the subfilters $F_{a}, F_{1}, F_{2}, F_{m a}$ and $F_{m c}$, respectively, such that the objective function (2) is minimized. The objective function is highly nonlinear with respect to the coefficients of the subfilters. Thus, it is very difficult to minimize the objective function using any direct optimization approach. In [21], an unconstrained WLS algorithm is used to jointly optimize the subfilters of an FIR filter synthesized using the original one-stage FRM structure. In each step, a quasi-Newton method is employed, where the Davidon-Fletcher-Powell (DFP) formula is used to update the search direction. The objective function is highly nonlinear and nonconvex with respect to the coefficients. Furthermore, its Hessian matrix is dense. Thus, the method can be computationally expensive for large problems. The optimization problem addressed in [21] is for the one-stage FRM structure. 
In contrast, the optimization problem described in this paper is for the modified FRM structure, where the single band-edge shaping filter in the one-stage FRM structure is replaced by a cascade combination of three subfilters. Clearly, the optimization problem addressed in this paper is much more complicated than that considered in [21]. Therefore, the direct optimization method proposed in [21] is unlikely to work for this problem.

By careful examination of Problem $(P)$, we see that it can be conveniently decomposed into several subproblems, as certain subsets of $\left\{\boldsymbol{h}_{a}, \boldsymbol{h}_{1}, \boldsymbol{h}_{2}, \boldsymbol{h}_{m a}, \boldsymbol{h}_{m c}\right\}$ affect fairly distinct parts of the frequency range [1]. In the part of the frequency range where $\boldsymbol{h}_{m a}$ and $\boldsymbol{h}_{m c}$ are both equal to 1 or 0 , the error is determined mainly by $\boldsymbol{h}_{m a}$ and $\boldsymbol{h}_{m c}$, while the effect of $\left\{\boldsymbol{h}_{a}, \boldsymbol{h}_{1}, \boldsymbol{h}_{2}\right\}$ is of secondary importance. On the other hand, in the frequency range where $\left|\boldsymbol{h}_{m a}(w)-\boldsymbol{h}_{m c}(w)\right|$ is large, (i.e., when $w \approx w_{p}$ or $w \approx w_{s}$ ), the error is very much determined by $\left\{\boldsymbol{h}_{a}, \boldsymbol{h}_{1}, \boldsymbol{h}_{2}\right\}$. We thus divide $\left\{\boldsymbol{h}_{a}, \boldsymbol{h}_{1}, \boldsymbol{h}_{2}, \boldsymbol{h}_{m a}, \boldsymbol{h}_{m c}\right\}$ into two subsets, $\left\{\boldsymbol{h}_{a}, \boldsymbol{h}_{1}, \boldsymbol{h}_{2}\right\}$ and $\left\{\boldsymbol{h}_{m a}, \boldsymbol{h}_{m c}\right\}$. The influence of each subset is largely restricted to the corresponding parts of the frequency range. Their effects are mutually compensated only on relatively small intervals of the frequency range. We therefore propose an alternating variable approach based on these two groups of coefficients to solve Problem $(P)$. For this, let us first define the following two optimization subproblems.

Problem $\left(P_{1}\right)$ : The coefficients $\boldsymbol{h}_{a}, \boldsymbol{h}_{1}$ and $\boldsymbol{h}_{2}$ of the objective function (2) are taken as fixed. Let the corresponding new objective function be denoted as $\epsilon_{1}\left(\left(\boldsymbol{h}_{m a}, \boldsymbol{h}_{m c}\right) \mid B, \boldsymbol{h}_{a}, \boldsymbol{h}_{1}, \boldsymbol{h}_{2}\right)$, where $B$ is the weighting function which is to be appropriately updated.

Problem $\left(P_{2}\right)$ : The coefficients $\boldsymbol{h}_{m a}$ and $\boldsymbol{h}_{m c}$ of the objective function (2) are taken as fixed. Let the corresponding new objective function be denoted as $\epsilon_{2}\left(\left(\boldsymbol{h}_{a}, \boldsymbol{h}_{1}, \boldsymbol{h}_{2}\right) \mid B, \boldsymbol{h}_{m a}, \boldsymbol{h}_{m c}\right)$, where $B$ is the weighting function which is to be appropriately updated.

Problem $(P)$ : is solved through solving Problem $\left(P_{1}\right)$ and Problem $\left(P_{2}\right)$ alternately. Problems $\left(P_{1}\right)$ and $\left(P_{2}\right)$ are solved as follows.

Note that Problem $\left(P_{1}\right)$ contains two unknown sets of coefficients $\boldsymbol{h}_{m a}$ and $\boldsymbol{h}_{m c}$. These belong to the subfilters $F_{m a}$ and $F_{m c}$, respectively, which are connected in parallel. As a result, the frequency response magnitude of the overall filter $F(w)$ is linear with respect to the coefficients in $\boldsymbol{h}_{m a}$ and $\boldsymbol{h}_{m c}$. Therefore, Problem $\left(P_{1}\right)$ can be solved analytically (details are given in Section III-A). Problem $\left(P_{2}\right)$ contains three unknown sets of coefficients, $\boldsymbol{h}_{a}, \boldsymbol{h}_{1}$ and $\boldsymbol{h}_{2}$ belonging to the subfilters, $F_{a}, F_{1}$ and $F_{2}$, respectively. These subfilters are connected in a cascade fashion. Thus, the frequency response magnitude of the overall filter $F(w)$ is nonlinear with respect to the coefficients, $\boldsymbol{h}_{a}, \boldsymbol{h}_{1}$ and $\boldsymbol{h}_{2}$. To overcome the complexity in the minimization on the objective function (2) with respect to the coefficients, $\boldsymbol{h}_{a}, \boldsymbol{h}_{1}$ and $\boldsymbol{h}_{2}$, we decompose Problem $\left(P_{2}\right)$ further into three subproblems and then use an alternating variable method to solve it. Let us define the following three optimization subproblems.

Problem $\left(P_{21}\right)$ : The coefficients $\boldsymbol{h}_{1}, \boldsymbol{h}_{2}, \boldsymbol{h}_{m a}$ and $\boldsymbol{h}_{m c}$ of the objective function (2) are taken as fixed. Let the corresponding new objective function be denoted as $\epsilon_{21}\left(\boldsymbol{h}_{a} \mid B, \boldsymbol{h}_{1}, \boldsymbol{h}_{2}, \boldsymbol{h}_{m a}, \boldsymbol{h}_{m c}\right)$, where $B$ is the weighting function which is to be appropriately updated.
Problem $\left(P_{22}\right)$ : The coefficients $\boldsymbol{h}_{a}, \boldsymbol{h}_{2}, \boldsymbol{h}_{m a}$ and $\boldsymbol{h}_{m c}$ of the objective function (2) are taken as fixed. Let the corresponding new objective function be denoted as $\epsilon_{22}\left(\boldsymbol{h}_{1} \mid B, \boldsymbol{h}_{a}, \boldsymbol{h}_{2}, \boldsymbol{h}_{m a}, \boldsymbol{h}_{m c}\right)$, where $B$ is the weighting function which is to be appropriately updated.

Problem ( $\left.P_{23}\right)$ : The coefficients $\boldsymbol{h}_{a}, \boldsymbol{h}_{1}, \boldsymbol{h}_{m a}$ and $\boldsymbol{h}_{m c}$ of the objective function (2) are taken as fixed. Let the corresponding new objective function be denoted as $\epsilon_{23}\left(\boldsymbol{h}_{2} \mid B, \boldsymbol{h}_{1}, \boldsymbol{h}_{2}, \boldsymbol{h}_{m a}, \boldsymbol{h}_{m c}\right)$, where $B$ is the weighting function which is to be appropriately updated.

Problem $\left(P_{2}\right)$ is then solved through solving a sequence of Problems $\left(P_{21}\right),\left(P_{22}\right)$ and $\left(P_{23}\right)$ alternately.

There are now two instances of an alternating variable search. The first one is to solve Problem $(P)$ via Problems $\left(P_{1}\right)$ and $\left(P_{2}\right)$. The second one is to solve Problem $\left(P_{2}\right)$ via Problems $\left(P_{21}\right),\left(P_{22}\right)$ and $\left(P_{23}\right)$. However, this approach is computationally time-consuming. Thus, we propose another technique, where the alternating search within Problem $\left(P_{2}\right)$ is incorporated into the alternating search between Problems $\left(P_{1}\right)$ and $\left(P_{2}\right)$. It may be described as follows:

Data: Choose a positive integer $m$, and a small positive real number $\Delta$.

Step 1) Find an initial filter $\left\{*, \boldsymbol{h}_{1}^{1}, \boldsymbol{h}_{2}^{1}, \boldsymbol{h}_{m a}^{1}, \boldsymbol{h}_{m c}^{1}\right\}$ (without $\boldsymbol{h}_{a}^{1}$ ) by designing the subfilters, $F_{1}(w), F_{2}(w), F_{m a}(w)$ and $F_{m c}(w)$, separately. Set the initial weighting function, $B_{1}(w)=1$, and iteration number, $k=1$. Set $i=0$ and $j=0$.

Step 2) Minimize $\epsilon_{21}\left(\boldsymbol{h}_{a} \mid B_{k}, \boldsymbol{h}_{1}^{k}, \boldsymbol{h}_{2}^{k}, \boldsymbol{h}_{m a}^{k}, \boldsymbol{h}_{m c}^{k}\right)$ with respect to the coefficients $\boldsymbol{h}_{a}$. Denote the optimal coefficients obtained as $h_{a}^{k}$.

Step 3) Minimize $\epsilon_{1}\left(\left(\boldsymbol{h}_{m a}, \boldsymbol{h}_{m c}\right) \mid B_{k}, \boldsymbol{h}_{a}^{k}, \boldsymbol{h}_{1}^{k}, \boldsymbol{h}_{2}^{k}\right)$ with respect to the coefficients $\boldsymbol{h}_{m a}$ and $\boldsymbol{h}_{m c}$. Denote the optimal coefficients obtained as $\boldsymbol{h}_{m a}^{k}$ and $\boldsymbol{h}_{m c}^{k}$. If $j=0$, goto Step 4. If $j=1$, goto Step 5. If $j=2$, set $i=i+1$. If $i=m$, goto Step 6. Otherwise, set $j=0$ and goto Step 2).

Step 4) Minimize $\epsilon_{22}\left(\boldsymbol{h}_{1} \mid B_{k}, \boldsymbol{h}_{a}^{k}, \boldsymbol{h}_{2}^{k}, \boldsymbol{h}_{m a}^{k}, \boldsymbol{h}_{m c}^{k}\right)$ with respect to the coefficients $\boldsymbol{h}_{1}$. Denote the optimal coefficients obtained as $\boldsymbol{h}_{1}^{k}$. Set $j=1$. Goto Step $3)$.

Step 5) Minimize $\epsilon_{23}\left(\boldsymbol{h}_{2} \mid B_{k}, \boldsymbol{h}_{a}^{k}, \boldsymbol{h}_{1}^{k}, \boldsymbol{h}_{m a}^{k}, \boldsymbol{h}_{m c}^{k}\right)$ with respect to the coefficients $\boldsymbol{h}_{2}$. Denote the optimal coefficients obtained as $\boldsymbol{h}_{2}^{k}$. Set $j=2$ and goto Step 3).

Step 6) Calculate $\delta^{k}=\max _{w \in\left[0, w_{p}\right] \cup\left[w_{s}, \pi\right]} W(w) \mid D(w)-$ $F(w) \mid$. If $\left|\delta^{k}-\delta^{k-1}\right|<\Delta$, where $\Delta$ is a prescribed tolerance, then stop. Otherwise, update $B_{k+1}(w)=$ $B_{k}(w) \overline{\bar{\beta}}_{k}(w)$, where $\overline{\bar{\beta}}_{k}(w)$ is defined in the next section. Set $k=k+1, i=0, j=0$ and go to Step 2).

From our extensive numerical simulation study, we observe that for the filter design using the above procedure, the filter lengths $l_{a}, l_{1}, l_{2}, l_{m a}, l_{m c}$ of the subfilters $F_{a}, F_{1}, F_{2}, F_{m a}$ and $F_{m c}$ are estimated to be approximately $100,100,85,65$ and 65 percent, respectively, of those designs obtained by the technique in [11]. 
Remark: In Step 6) of the algorithm, the weighting function is updated every $m$ iterations. From our extensive numerical simulation experience, we observe that it is sufficient to choose $m$ to be between 3 and 5 . The tolerance $\Delta$ determines the terminating condition for the algorithm.

\section{A. Solution of Problem $\left(P_{1}\right)$}

Note that the optimality condition for the coefficients $h_{m a}$ and $\boldsymbol{h}_{m c}$ of Problem $\left(P_{1}\right)$ is easily obtained by setting the gradient of the objective function $\epsilon_{1}$ with respect to these coefficients to zero. This gives rise to the following system of linear equations:

$$
R U \boldsymbol{X}=R \boldsymbol{H}
$$

where

$$
\begin{aligned}
U & =\left[\begin{array}{ccc}
U_{1}\left(w_{1}\right) & , & U_{2}\left(w_{1}\right) \\
U_{1}\left(w_{2}\right) & , & U_{2}\left(w_{2}\right) \\
\vdots & , & \vdots
\end{array}\right] \\
U_{1}(w) & =\left\{G_{1}(w) \operatorname{trig}_{m a}(w, 1), G_{1}(w) \operatorname{trig}_{m a}(w, 2), \ldots\right\} \\
U_{2}(w) & =\left\{G_{2}(w) \operatorname{trig}_{m c}(w, 1), G_{2}(w) \operatorname{trig}_{m c}(w, 2), \ldots\right\}
\end{aligned}
$$

where $\operatorname{trig}_{m a}(w, i)$ and $\operatorname{trig}_{m c}(w, i)$ are appropriate trigonometric functions for subfilters $F_{m a}(w)$ and $F_{m c}(w)$, respectively, and

$$
\begin{aligned}
\boldsymbol{X} & =\left\{h_{m a}(1), h_{m a}(2), \ldots, h_{m c}(1), h_{m c}(2), \ldots\right\}^{T} \\
\boldsymbol{H} & =\left\{D\left(w_{1}\right), D\left(w_{2}\right), \cdots\right\}^{T} .
\end{aligned}
$$

$R$ is the weighting matrix given by

$$
R=\left[\begin{array}{ccc}
B\left(w_{1}\right) & \bigcirc & \\
& B\left(w_{2}\right) & \\
\bigcirc & & \ddots
\end{array}\right]
$$

and $\left\{w_{i}\right\}$ is a dense set of frequencies uniformly distributed in $\left[0, w_{p}\right] \cup\left[w_{s}, \pi\right]$. The least-squares solution of (3) is given by

$$
\boldsymbol{X}=\left(U^{T} R^{2} U\right)^{-1} U^{T} R^{2} \boldsymbol{H} .
$$

\section{B. Solutions of Problems $\left(P_{21}\right),\left(P_{22}\right)$ and $\left(P_{23}\right)$}

Note that the optimality conditions for Problems $\left(P_{21}\right),\left(P_{22}\right)$, and $\left(P_{23}\right)$ are easily obtained by setting the gradients of the objective functions $\epsilon_{21}, \epsilon_{22}$ and $\epsilon_{23}$ with respect to their associated coefficients equal to zero. They give rise to the following form of linear system:

$$
R V \boldsymbol{Y}=R \boldsymbol{G}
$$

where

$$
\begin{aligned}
V & =\left[\begin{array}{ccc}
V_{1}\left(w_{1}\right), & V_{2}\left(w_{1}\right), & \ldots \\
V_{1}\left(w_{2}\right), & V_{2}\left(w_{2}\right), & \ldots \\
\vdots & \vdots & \ddots
\end{array}\right] \\
\boldsymbol{Y} & =\{Y(1), Y(2), \ldots\}^{T} \\
& =\left\{g\left(w_{1}\right), g\left(w_{2}\right), \ldots\right\}^{T}
\end{aligned}
$$

and the weighting matrix $R$ is defined by (7).
The least-squares solution of (9) is given by

$$
\boldsymbol{Y}=\left(V^{T} R^{2} V\right)^{-1} V^{T} R^{2} G .
$$

For Problem $\left(P_{21}\right), V_{i}(w)=\left(F_{m a}(w)-\right.$ $\left.F_{m c}(w)\right) F_{1}(w) F_{2}(w) \operatorname{trig}_{a}\left(M_{a} w, i\right), \quad$ where $\operatorname{trig}_{a}(w, i)$ are appropriate trigonometric functions for subfilter $F_{a}(w)$, $Y(i)=h_{a}(i)$, and $g(w)=F_{m c}(w)+F_{2}(w)\left(F_{m a}(w)-\right.$ $\left.F_{m c}(w)\right)-D(w)$. For Problem $\left(P_{22}\right), V_{i}(w)=$ $\left(F_{m a}(w) \quad-\quad F_{m c}(w)\right) F_{a}(w) F_{2}(w) \operatorname{trig}_{1}\left(M_{1} w, i\right)$, where $\operatorname{trig}_{1}(w, i)$ are appropriate trigonometric functions for subfilter $F_{1}(w), Y(i)=h_{1}(i)$, and $g(w)=F_{m c}(w)+F_{2}(w)\left(F_{m a}(w)-F_{m c}(w)\right)-D(w)$.

For Problem $\left(P_{23}\right), V_{i}(w)=\left(F_{m a}(w)-F_{m c}(w)\right)(1.0-$ $\left.F_{a}(w) F_{1}(w)\right) \operatorname{trig}_{2}\left(M_{2} w, i\right)$, where $\operatorname{trig}_{2}(w, i)$ are appropriate trigonometric functions for subfilter $F_{2}(w), Y(i)=h_{2}(i)$, and $g(w)=F_{m c}(w)-D(w)$.

The updated error weighting function at each iteration $k$ is given [22] by $B_{k+1}\left(w_{n}\right)=B_{k}\left(w_{n}\right) \beta_{k}\left(w_{n}\right)$

$$
\text { with } \quad \beta_{k}\left(w_{n}\right)=\frac{\left|E_{k}\left(w_{n}\right)\right|}{\sum_{m} B_{k}\left(w_{m}\right)\left|E_{k}\left(w_{m}\right)\right|} .
$$

In order to prevent $\beta_{k}\left(w_{n}\right)$ from getting too close to zero, we introduce a lower bound, $\gamma$, similar to the one used in [22]

$$
\begin{aligned}
\bar{\beta}_{k}(w) & = \begin{cases}\beta_{k}(w) & \text { if } \beta_{k}(w) \geq \gamma \\
\gamma & \text { otherwise, }\end{cases} \\
\text { where } \quad \gamma \leq v & =\frac{\min _{\left\{\boldsymbol{h}_{a}, \ldots, \boldsymbol{h}_{m c}\right\}} \max _{w \in\left[0, w_{p}\right] \cup\left[w_{s}, \pi\right]} E(w)}{\sum_{m} B_{k}\left(w_{m}\right)\left|E_{k}\left(w_{m}\right)\right|} .
\end{aligned}
$$

To accelerate the convergence, $\bar{\beta}_{k}\left(w_{n}\right)$ is made proportional to a higher power of $\bar{\beta}_{k}\left(w_{n}\right)$ as given by [22]

$$
\overline{\bar{\beta}}_{k}\left(w_{n}\right)=\left(A_{k} \frac{\overline{\beta_{k}}\left(w_{n}\right)}{W\left(w_{n}\right)}\right)^{\theta} .
$$

Here, $W(w)$ is the minimax weighting function as indicated before, and $A_{k}$ is a constant which is selected to maintain a reasonable dynamic range for the value of $B_{k}\left(w_{n}\right)$. As in [22], the constant $A_{k}$ may be selected as the average value of $D\left(w_{n}\right) / B_{k}\left(w_{n}\right)$ over all $n$. The constant $\theta$ affects the rate of convergence, see [22] for details. We take the value of $\theta$ to be between 1 and 1.7 as in [22].

\section{DESIGn EXAMPLE}

As in [11] and [12], a linear phase low-pass FIR filter with the passband frequencies $0 \leq w / 2 \pi \leq 0.3$ and the stopband frequencies $0.301 \leq w / 2 \pi \leq 0.5$ is considered. The allowable maximum passband and stopband ripples are $\delta_{p}=0.01$ and $\delta_{s}=0.0001$, respectively. We use the same interpolation ratios as in [11]: $M_{a}=39, M_{1}=13$, and $M_{2}=4$. For WLS Chebyshev solution, we use 10001 sample points in the frequency range. Our method takes 364 iterations to meet the minimax criteria with Problems $\left(P_{1}\right),\left(P_{21}\right),\left(P_{1}\right),\left(P_{22}\right),\left(P_{1}\right)$, and $\left(P_{23}\right)$ being solved alternately 4 times at each iteration. All computations are performed on an SGI Origin 2000. The computer used 13.1 minutes to complete the calculation. The filter lengths of $F_{a}, F_{1}, F_{2}, F_{m a}$, and $F_{m c}$ used are 83, 21, 49, 38, and 16 , respectively. 105 multipliers are needed. The maximum 


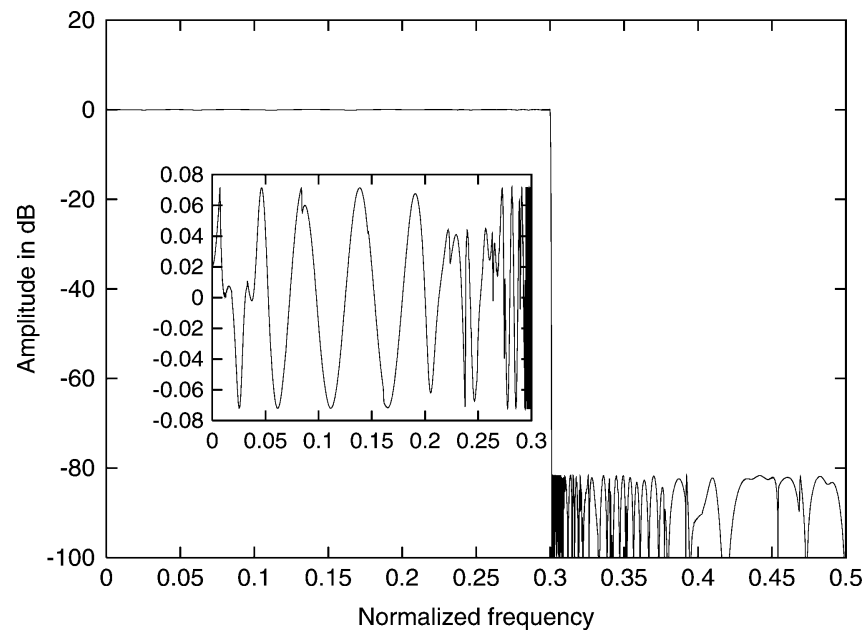

Fig. 4. Frequency response of the overall filter.

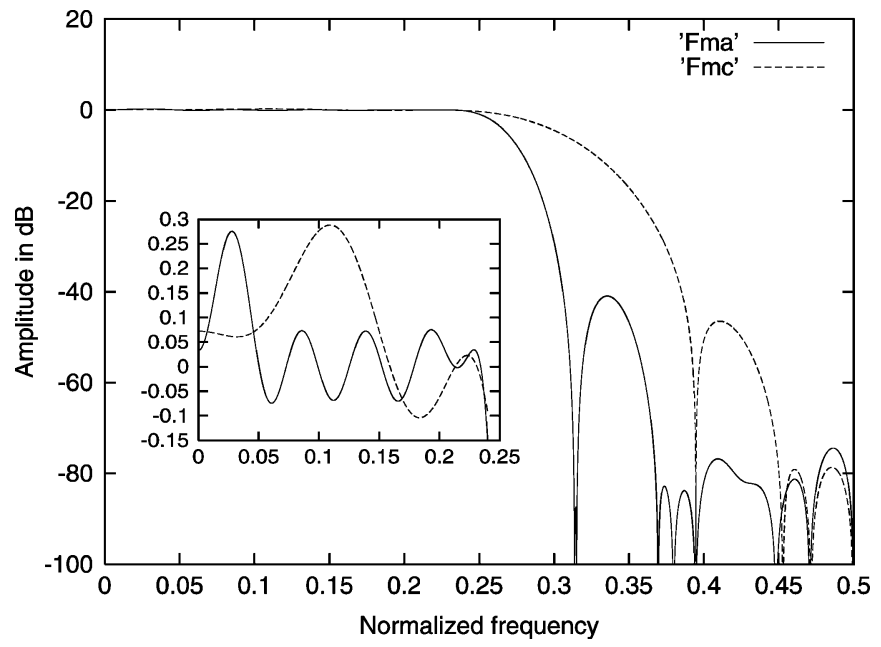

Fig. 5. Frequency response of $F_{m a}(w)$ and $F_{m c}(w)$.

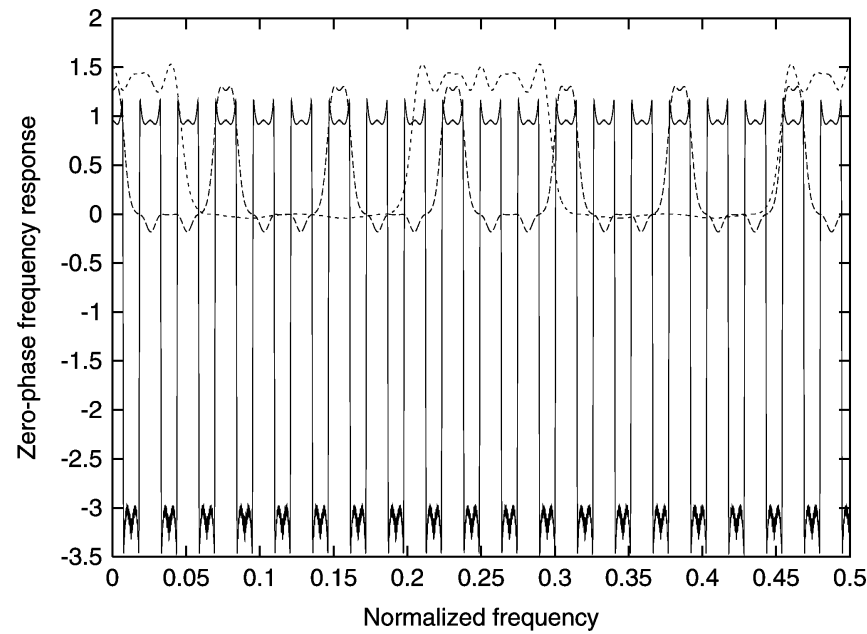

Fig. 6. Frequency responses of $F_{a}\left(M_{a} w\right)$ (solid line), $F_{1}\left(M_{1} w\right)$ (dashed line) and $F_{2}\left(M_{2} w\right)$ (doted line).

passband and stopband ripples achieved are $\delta_{p}=0.00835$ and $\delta_{s}=0.0000835$, respectively. For comparison, the filter lengths of $F_{a}, F_{1}, F_{2}, F_{m a}$, and $F_{m c}$ used in [11] to meet the requirements are $83,21,57,56,24$, respectively. 122 multipliers are needed. The filter designed by using our approach yields a
$14 \%$ savings in the number of multipliers required when compared with the filter produced in [11]. The frequency response of the filter and subfilters designed by the proposed method are depicted in Figs. 4- 6, respectively.

\section{REFERENCES}

[1] Y. C. Lim, "Frequency-response masking approach for the synthesis of sharp linear phase digital filter," IEEE Trans. Circuits Syst., vol. CAS-33, no. 4, pp. 357-364, Apr. 1986.

[2] Y. C. Lim and Y. Lian, "The optimum design of one- and two-dimensional FIR filters using the frequency response masking technique," IEEE Trans. Circuits Syst. II, Analog Digit. Signal Process., vol. 40, no. 2, pp. 88-95, Feb. 1993.

[3] Y. C. Lim and S. H. Low, "Frequency-response masking approach for the synthesis of sharp two-dimensional diamond-shaped filters," IEEE Trans. Circuits Syst. II, Analog Digit. Signal Process., vol. 45, no. 12, pp. 1573-1584, Dec. 1998.

[4] T. Saramäki and Y. C. Lim, "Use of the Remez algorithm for designing FRM based FIR filters," Circuits, Syst. Signal Process., vol. 22, no. 2, pp. 77-97, Apr. 2003

[5] M. G. Bellanger, "Improved design of long FIR filters using the frequency masking technique," in Proc. IEEE Int. Conf. Acoust., Speech, Signal Process., Atlanta, GA, May 1996, pp. 1272-1275.

[6] Y. Lian, "A new frequency response masking structure with reduced complexity for FIR filter design," in Proc. IEEE Int. Symp. Circuits Syst., vol. II, Sydney, NSW, Australia, May 2001, pp. 609-612.

[7] — " "Reducing the complexity of the masking filter in a frequency-response masking approach," in Proc. 3rd Int. Conf. Inf., Commun., Signal Process., Singapore, Oct. 2001.

[8] _ _ "A modified frequency-response masking structure for high-speed FPGA implementation of sharp FIR filters," J. Circuits Syst. Comp., vol. 12, no. 5, pp. 643-654, Oct. 2003.

[9] — - "Complexity reduction for FRM-based FIR filters using the prefilter-equalizer technique," Circuits, Syst. Signal Process., vol. 22, no. 2, pp. 137-155, Apr. 2003.

[10] Y. Lian and Y. C. Lim, "Reducing the complexity of frequency-response masking filters using half band filters," Signal Process., vol. 42, no. 3 , pp. 227-230, Mar. 1995

[11] Y. Lian and C. Z. Yang, "Complexity reduction by decoupling the masking filters from the bandedge shaping filter in the FRM technique," Circuits, Syst. Signal Process., vol. 22, no. 2, pp. 115-135, Apr. 2003.

[12] Y. Lian, L. Zhang, and C. C. Ko, "An improved frequency response masking approach designing sharp FIR filters," Signal Proces., vol. 81, pp. 2573-2581, Dec. 2001.

[13] Y. C. Lim and Y. Lian, "Frequency response masking approach for digital filter design: Complexity reduction via masking filter factorization,' IEEE Trans. Circuits Syst. II, Analog Digit. Signal Process., vol. 41, no. 8, pp. 518-525, Aug. 1994.

[14] T. Saramäki and J. Yli-Kaakinen, "Optimization of frequency-response masking based FIR filters with reduced complexity," in Proc. IEEE Int Symp. Circuits Syst., vol. III, Phoenix, AZ, May 2002, pp. 225-228.

[15] R. Yang, B. Liu, and Y. C. Lim, "A new structure of sharp transition FIR filters using frequency-response masking," IEEE Trans. Circuits Syst., vol. CAS-35, no. 8, pp. 955-966, Aug. 1988.

[16] L. Zhang, Y. Lian, and C. C. Ko, "A new approach for designing sharp FIR filters using frequency-response masking technique," Proc. 9th IEEE DSP Workshop, Oct. 2000.

[17] Y. Neuvo, C. Y. Dong, and S. K. Mitra, "Interpolated finite impulse response filters," IEEE Trans. Acoust. Speech, Signal Process, vol. ASSP-32, no. 3, pp. 563-570, Jun. 1984.

[18] J. Cabezas and P. Diniz, "FIR filters using interpolated prefilters and equalizers," IEEE Trans. Circuits Syst., vol. 37, no. 1, pp. 17-23, Jan. 1990.

[19] T. Saramäki, Y. Neuvo, and S. K. Mitra, "Design of computationally efficient interpolated FIR filters," IEEE Trans. Circuits Syst., vol. 35, no. 1 , pp. $70-88$, Jan. 1988

[20] T. Saramäki and H. Johansson, "Optimization of FIR filters using the frequency-response masking approach," in Proc. IEEE Int. Symp. Cir cuits Syst., vol. II, Sydney, NSW, Australia, May 2001, pp. 177-180.

[21] Y. J. Yu and Y. C. Lim, "FRM based FIR filter design-The WLS approach," in Proc. IEEE Int. Symp. Circuits Syst., vol. II, Phoenix, AZ, May 2002, pp. 221-224.

[22] Y. C. Lim, J. H. Lee, C. K. Chen, and R. H. Yang, "A weighted least squares algorithm for quasi-equiripple FIR and IIR digital filter design," IEEE Trans. Signal Proces., vol. 40, no. 3, pp. 551-558, Mar. 1992. 